\title{
Predicting potential survival benefit of renal transplantation in patients with chronic kidney disease
}

\author{
Carl van Walraven MD MSc, Peter C. Austin PhD, Greg Knoll MD MSc
}

Previously published at www.cmaj.ca

\section{ABSTRACT}

Background: To facilitate decision-making about treatment options for patients with end-stage renal disease considering kidney transplantation, we sought to develop an index for clinical prediction of risk for death.

Methods: We derived and validated a multivariable survival model predicting time to death in 169393 patients with endstage renal disease who were eligible for transplantation. We modified the model into a simple point-system index.

Results: Deaths occurred in $23.5 \%$ of the cohort. Twelve variables independently predicted death: age, race, cause of kidney failure, body mass index, comorbid disease, smoking, employment status, serum albumin level, year of first renal replacement therapy, kidney transplantation, time to transplant wait-listing and time on the wait list. The index separated patients into 26 groups having significantly unique five-year survival, ranging from $97.8 \%$ in the lowest-risk group to $24.7 \%$ in the highest-risk group. The index score was discriminative, with a concordance probability of 0.746 (95\% Cl 0.741-0.751). Observed survival in the derivation and validation cohorts was similar for each level of index score in $93.9 \%$ of patients.

Interpretation: Our prognostic index uses commonly available information to predict mortality accurately in patients with end-stage renal disease. This index could provide valuable quantitative data on survival for clinicians and patients to use when deciding whether to pursue transplantation or remain on dialysis.

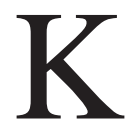
idney transplantation, which improves healthrelated quality of life and survival compared with dialysis, is the treatment of choice for end-stage renal disease. ${ }^{1-3}$ Overall, recipients of kidney transplantation have a $68 \%$ lower risk of death compared with patients eligible for transplantation who remain on dialysis. ${ }^{1}$ In subgroup analyses of broad patient categories (including sex, - cause of renal failure and diabetic status), patients in all categories were found to have benefited from transplantation.

These data inform patients about the overall risks and benefits of kidney transplantation. However, prognostication in renal failure remains difficult at the individual level. Models have been developed to predict survival after kidney transplantation. ${ }^{4-7}$ However, these models have not been widely used, because they lack a simple point system to determine individual survival, provide no comparison to the alternative treatment of staying on dialysis, exclude the option of livingdonor transplantation, or require detailed information on the donor that is not known at the time of wait-listing. ${ }^{4-7}$

We aimed to derive and validate a new index to quantify survival accurately for the various treatment options facing a patient with end-stage renal disease. We based this prognostic index on readily available data, so that it could be easily implemented in the clinical setting when transplantationrelated counseling takes place. We modified this model into a simple scoring system to quantify survival without transplantation, with deceased-donor transplantation or with livingdonor transplantation. Our goal was to improve decisionmaking by patients and physicians by providing quantitative information about survival at the time of transplantationrelated counseling.

\section{Methods}

We used data from the United States Renal Data System, a national data-reporting system that captures information on all American patients with end-stage renal disease who receive renal replacement therapy. Reporting to the United States Renal Data System is mandatory for all centres that treat such patients and is required for payment of treatmentrelated costs by Medicare and Medicaid Services.

Our study was approved by the Ottawa Hospital Research Ethics Board.

\section{Inclusion criteria}

We included all patients who had been placed on the renal transplant wait list from January 1995 to October 2006, as well as those who had received a kidney transplant as their first renal replacement therapy (i.e., pre-emptive transplantation) during this same period. We chose 1995 as the study's start date to coincide with the introduction of a new form for recording patient information at baseline. The end-date of the

From the Faculty of Medicine (van Walraven, Knoll), University of Ottawa; the Ottawa Health Research Institute (van Walraven, Knoll), Ottawa, Ont.; and the Institute for Clinical Evaluative Sciences (van Walraven, Austin), Toronto, Ont.

CMAJ 2010. DOI:10.1503/cmaj.091661 
study was 2006 because that was the final year for which data were available.

We excluded patients for whom data were missing about date of birth, date of first renal replacement therapy or date of transplant wait-listing. We also excluded patients who were not between ages 18 and 100 years and those who had undergone renal transplantation before the study's start-date. Finally, we did not include renal transplantations that were part of multipleorgan procedures (e.g., kidney-pancreas transplantation).

\section{Derivation and validation models}

We divided patients randomly into equally sized derivation and validation groups. We used Cox regression analyses to determine the independent association between potentially prognostic covariates and time to death. Observation of patients started when they were placed on the renal transplantation list. For pre-emptive transplantations, observation was started on the day of transplantation. Observation was ended when patients died of any cause or was censored at the study's end (Sept. 31, 2006). The variables in the model are listed in Table 1; they include year of first renal replacement therapy and number of years from first renal replacement therapy to transplant wait-listing. Living-donor and deceased-donor renal transplantations were modelled as separately defined, timedependent covariates, as was number of years from listing to transplantation. All other covariates were fixed.

Because our study was focused on measuring the influence of transplantation on survival, we limited interactions in the model to those involving transplantation. We used fractional polynomial functions to determine the best linear or nonlinear form for continuous variables. ${ }^{8-10}$ To deal with missing data, we imputed values using Monte Carlo Markov chain methodology with PROC MI with five imputations. This Monte Carlo model used all of the variables that were examined in the multivariate model. Final parameter estimates and $\chi^{2}$ statistics for each covariate were determined with PROC MIANALYZE. Only covariates with $p<0.05$ were kept in the model.

Details of the creation and assessment of the index are available in Appendix 1, available at www.cmaj.ca/cgi/content /full/cmaj.091661/DC1.

\section{Results}

Between 1995 and 2006, 1709267 patients were entered in the United States Renal Data System for a first renal replacement therapy. We excluded 1539874 patients. Of these, $1511626(98.2 \%)$ had never been placed on the transplant wait list, $20003(1.3 \%)$ were entered in the data system only after failure of a first renal transplantation, $8080(0.52 \%)$ were not between ages 18 and 100 years, $91(0.01 \%)$ had an invalid date of first renal replacement therapy, and 74 $(0.001 \%)$ had an invalid date of birth. The remaining patients in the study totalled 169393 , with 84724 in the derivation group and 84669 in the validation group.

\section{Characteristics of participants}

The characteristics of study participants (Table 1) in the derivation group and those of participants in the validation group were nearly identical. The prevalence of missing information varied from $2.6 \%$ for information on comorbidities to $27.6 \%$ for information on serum albumin level. Median observation time was 3.6 years (interquartile range 1.7-6.4 years; total person-years of observation 714771 ). Death occurred in $23.5 \%$ of the cohort, with an annual death rate of $5.6 \%$. Overall, $55.9 \%$ of the cohort underwent transplantation (deceased-donor transplantation 38.7\%, living-donor 17.2\%).

\section{Regression model}

All of the variables in Table 1 were included in the regression model. Those that were independently associated with survival are listed in Table 2 and in Appendices 2 and 3 (available at www.cmaj.ca/cgi/content/full/cmaj.091661/DC1). Baseline type of renal disease, medical history, smoking status, employment status and race were independently associated with time to death (Table 2). Diabetes requiring insulin, chronic obstructive pulmonary disease, nonambulatory status and current smoking were the comorbidities with the strongest detrimental effect on survival, with adjusted hazard ratios close to 1.3 (Table 2). Protective factors included having polycystic kidney disease as the cause of renal failure (adjusted hazard ratio [HR] 0.59) or being of Asian, Spanish-American, or NativeAmerican ethnicity (adjusted HR 0.69). Patients of black ethnicity also survived longer than those of white ethnicity, but only in the absence of transplantation (Table 2).

The adjusted association of continuous variables with survival is presented in Appendix 2. Of all continuous factors, the association between age and death was the strongest, with a mortality risk that increased as patients aged, especially among those who underwent transplantation. Survival rates in all groups improved as serum albumin levels increased. Survival rate improved as the year of first renal transplantation increased, except for patients undergoing deceased-donor transplantation. Finally, delay from first renal replacement therapy to transplant wait-listing or delay to receipt of a pre-emptive transplant was associated with worse survival rates in all groups, most prominently among recipients of deceased-donor transplantation. The final regression model is presented in Appendix 3.

\section{Prognostic index}

The final prognostic index variables, along with the scoring system, are presented in Figure 1. Potential index scores ranged from -47 to +58 , with lower scores associated with better survival. The points assigned for patients' age, ethnicity, year of first renal replacement therapy, and time required to be entered on the transplant wait-list varied by transplant status. Points for all other variables were the same regardless of transplant status.

An individual patient's index score can be calculated by summing up the points for each applicable factor in Figure 1. For example, a transplant-nonrecipient, 45 -year-old ( +7 points), obese (body mass index [BMI] $40=0$ points) man of black ethnicity ( -4 points) with a serum albumin level of $35 \mathrm{~g} / \mathrm{L}(+5$ points) who has end-stage renal failure from glomerulonephritis ( -4 points) in 2001 ( -4 points) and is entered on the transplant list three months after his first renal replacement therapy (+4 points) has an index score of 4 , with an expected risk of death in 
Table 1: Characteristics of patients in study cohort

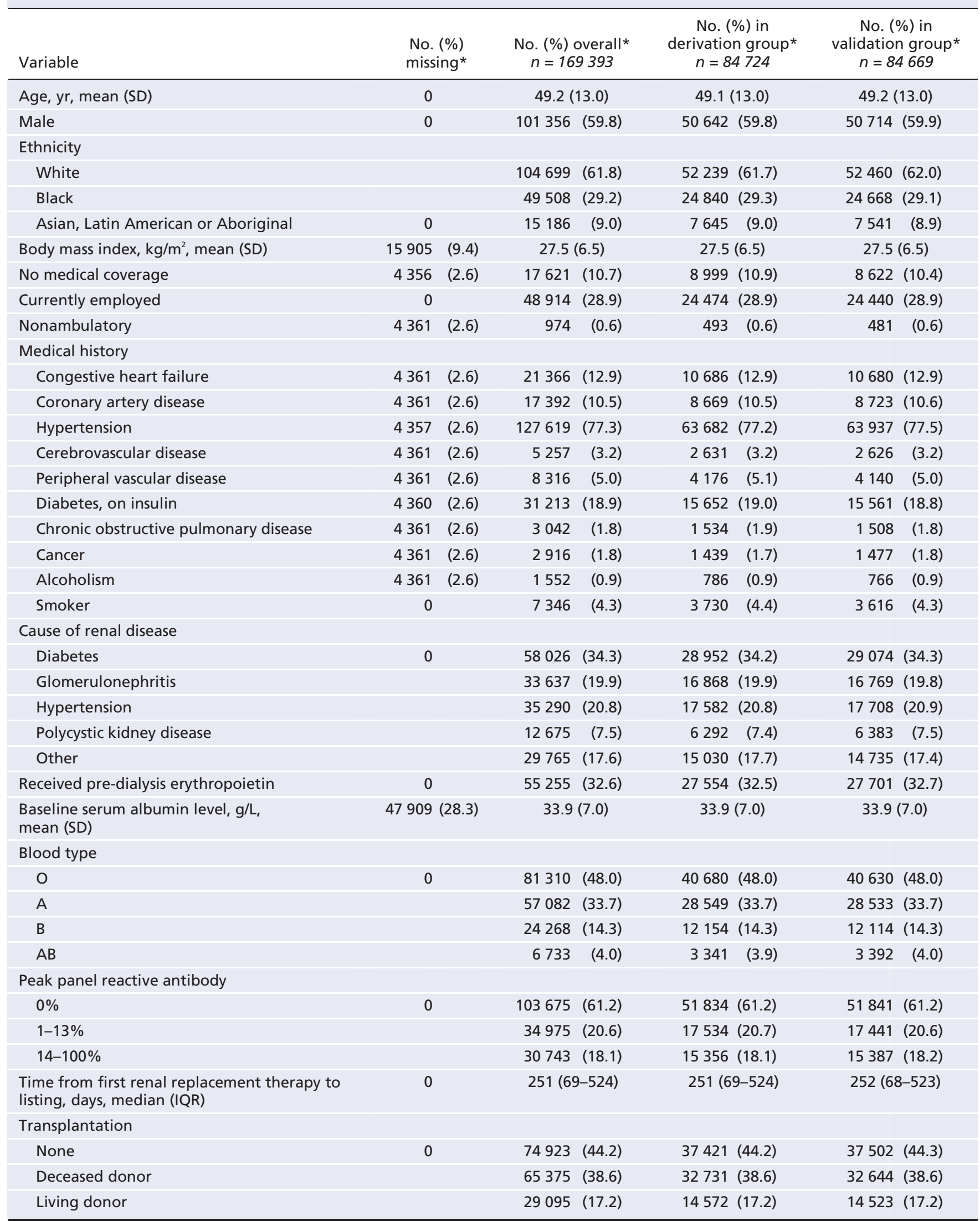

*Unless stated otherwise. 
five years of $18.2 \%$. If this patient received a living-donor transplantation nine months after being entered on the transplant wait-list, his index score would decrease to -13 points (age +10 , $\mathrm{BMI}+0$, black ethnicity -1 , serum albumin level +5 , glomerulonephritis -4 , year $2001-4$, delay of three months before listing +6 , transplantation -26 , years to transplantation $0.75+1$ ), with expected risk of death in five years decreasing to $4.8 \%$.

The prognostic index was highly discriminative for patient survival. Each index-score grouping identified in the derivation set remained significantly distinct from all other groups in the validation set (Appendix 4, available at www.cmaj.ca/cgi /content/full/cmaj.091661/DC1). Patients with higher scores always had shorter survival. Of the 26 index-score groupings displayed in Appendix 4, all but four of them contained three or fewer discrete index scores. The concordance probability of the index in the validation group was 0.746 (95\% confidence interval $[\mathrm{CI}]$ 0.741-0.751). This value indicates that, for all possible patient pairings, the patient with the greater predicted survival actually lived longer $74.6 \%$ of the time.

The expected and observed mortality for each prognostic index score are presented in Figure 2 and in Appendix 5 (available at www.cmaj.ca/cgi/content/full/cmaj.091661/DC1). Below a score of 13, the model-generated five-year expected risk of death was within the $95 \%$ CIs of the validation set for all index scores, except for several scores for which the expected survival rate significantly exceeded the observed survival rate. Between index scores of 14 and 22, the expected mortality risk underestimated the observed mortality by a mean of $4.8 \%$. At index scores above 30, the expected mortality tended to exceed the observed mortality. Observed rates were similar for each level of index score between the derivation and validation groups in $93.9 \%$ of all patients (Appendix 5).

\section{Interpretation}

We derived and internally validated an index that predicts overall survival in patients with end-stage renal disease who are eligible for transplantation. This prognostic index uses objective, readily available information to estimate the risk of death at five years.

This prognostic index will help patients and physicians make decisions related to renal replacement therapy by quantifying individual, expected survival with each treatment. Our index will allow clinicians to easily calculate the expected survival for an individual patient with and without kidney transplantation. Clinicians can use these quantitative data to counsel patients in the clinical setting about the expected benefits of deceased-donor or living-donor transplantation compared to those of remaining on dialysis. While the benefit of transplantation will be positive for most patients, the relative benefit of transplantation for some individual patients will be blunted. For such individuals, factors other than survival (e.g., quality of life, adverse effects of immunosuppressive medication, risk of malignancy and need for an extensive medical evaluation or ongoing re-evaluation) will become important issues to consider before proceeding with transplantation. ${ }^{2,11-15}$

Our prognostic index identified several factors whose significant influence on patient survival changed notably with transplant status. The detrimental effect of increasing patient age on survival was accentuated with transplantation. This effect is likely attributable to an increased risk of death among elderly patients in the early post-transplantation period. ${ }^{1.16}$ The detrimental effect of a delay from first renal replacement therapy to wait-listing for transplantation was amplified among recipients of living-donor transplantation and, more so, among those of deceased-donor transplantation. This finding is likely attributable to the known negative association between time on dialysis and patient survival after transplantation. ${ }^{17}$ Overall, our findings are very consistent with previous analyses of factors associated with survival in end-stage renal failure. ${ }^{18,19}$

The negative influence of transplantation on the interaction variables noted above was almost always greater in recipients of deceased-donor transplantation compared with livingdonor transplant recipients. This influence explains the initially unusual finding that both deceased- and living-donor transplantation had the same beneficial effect on mortality (Figure 1). After considering points for the interacting variables, we observed that patients who underwent deceaseddonor transplantation almost invariably had higher index scores and worse expected survival compared with patients who underwent living-donor transplantation.

Table 2: Factors independently associated with death among patients awaiting renal transplantation

\begin{tabular}{|c|c|}
\hline Variable & $\begin{array}{l}\text { Adjusted hazard rati } \\
(95 \% \mathrm{Cl})^{*}\end{array}$ \\
\hline \multicolumn{2}{|l|}{ Renal disease at baseline } \\
\hline Diabetes mellitus & $1.31(1.25-1.37)$ \\
\hline Hypertension & $0.92(0.88-0.97)$ \\
\hline Glomerulonephritis & $0.71(0.67-0.75)$ \\
\hline Polycystic kidney disease & $0.59(0.55-0.64)$ \\
\hline \multicolumn{2}{|l|}{ Medical history } \\
\hline $\begin{array}{l}\text { Chronic obstructive pulmonary } \\
\text { disease }\end{array}$ & $1.31(1.20-1.42)$ \\
\hline Nonambulatory & $1.27(1.10-1.47)$ \\
\hline Coronary artery disease & $1.18(1.13-1.23)$ \\
\hline Peripheral vascular disease & $1.17(1.11-1.24)$ \\
\hline Congestive heart failure & $1.17(1.12-1.21)$ \\
\hline Diabetes requiring insulin & $1.16(1.12-1.21)$ \\
\hline Cerebrovascular disease & $1.11(1.03-1.19)$ \\
\hline Hypertension & $0.91(0.88-0.94)$ \\
\hline Smoker & $1.30(1.22-1.38)$ \\
\hline Currently employed & $0.85(0.82-0.88)$ \\
\hline \multicolumn{2}{|l|}{ Ethnicity (v. white) } \\
\hline Asian, Latin American or Aboriginal & $0.69(0.65-0.73)$ \\
\hline \multicolumn{2}{|l|}{ Black } \\
\hline No transplant & $0.71(0.68-0.74)$ \\
\hline Deceased donor & $1.00(0.92-1.08)$ \\
\hline Living donor & $0.94(0.80-1.09)$ \\
\hline
\end{tabular}

Note: $\mathrm{Cl}=$ confidence interval.

*Each variable was adjusted for other variables in the table. 
Variable

Points*

Baseline serum albumin levelt

$<25$

25-27

28-32

33-37

38-39

40-41

$>41$

Body mass indext

$<20.4$

20.4-25.0

25.1-35.7

$>35.7$

Cause of renal failuret

Diabetes

Hypertension

Glomerulonephritis

Polycystic kidney disease

Other

Medical history

Chronic obstructive pulmonary disease

Nonambulatory

Congestive heart failure

Diabetes requiring insulin

Coronary artery disease

Peripheral vascular disease

Cerebrovascular disease

Hypertension

Smoker

Currently employed
Points* by transplantation status

\begin{tabular}{ccc}
\hline No & Deceased & Living \\
transplant & donor & donor
\end{tabular}

Age†

$<31$

31-37.5

$37.5-42.5$

$42.5-46.8$

46.8-50.3

50.3-53.7

$53.7-57.0$

$57.0-60.7$

$60.7-65.3$

$>65.3$

Ethnicity $\dagger$

White

Black

Other

Year of first renal replacement therapy†‡

$<1997$

1997

1998-1999

2000

2001

2002-2003

2004

$>2004$

$\begin{array}{lll}0 & 0 & 0\end{array}$

3

5

7

8

9

10

11

13

18

0

$-4$

$-4$

$$
5
$$

8

11

13

Time from first renal replacement therapy to listing, mot

$$
0
$$

$0.1-0.4$

0.5-3.6

3.7-5.9

6-8.3

8.4-11.2

$11.3-14.9$

15-20.6

20.7-31.6

31.7-75.1

Transplantation

Time from listing to transplantation, $\mathrm{yr}$

$$
\begin{aligned}
& <0.51 \\
& 0.51-1.92 \\
& 1.93-3.71 \\
& >3.71
\end{aligned}
$$

Figure 1: Prognostic variables and scoring system of index for the prediction of risk of death among patients with end-stage renal disease eligible for transplantation. *To calculate the index score of a patient, points for all factors that apply are summed. + Factor for which points are assigned to all patients. IIncludes pre-emptive transplantation. 


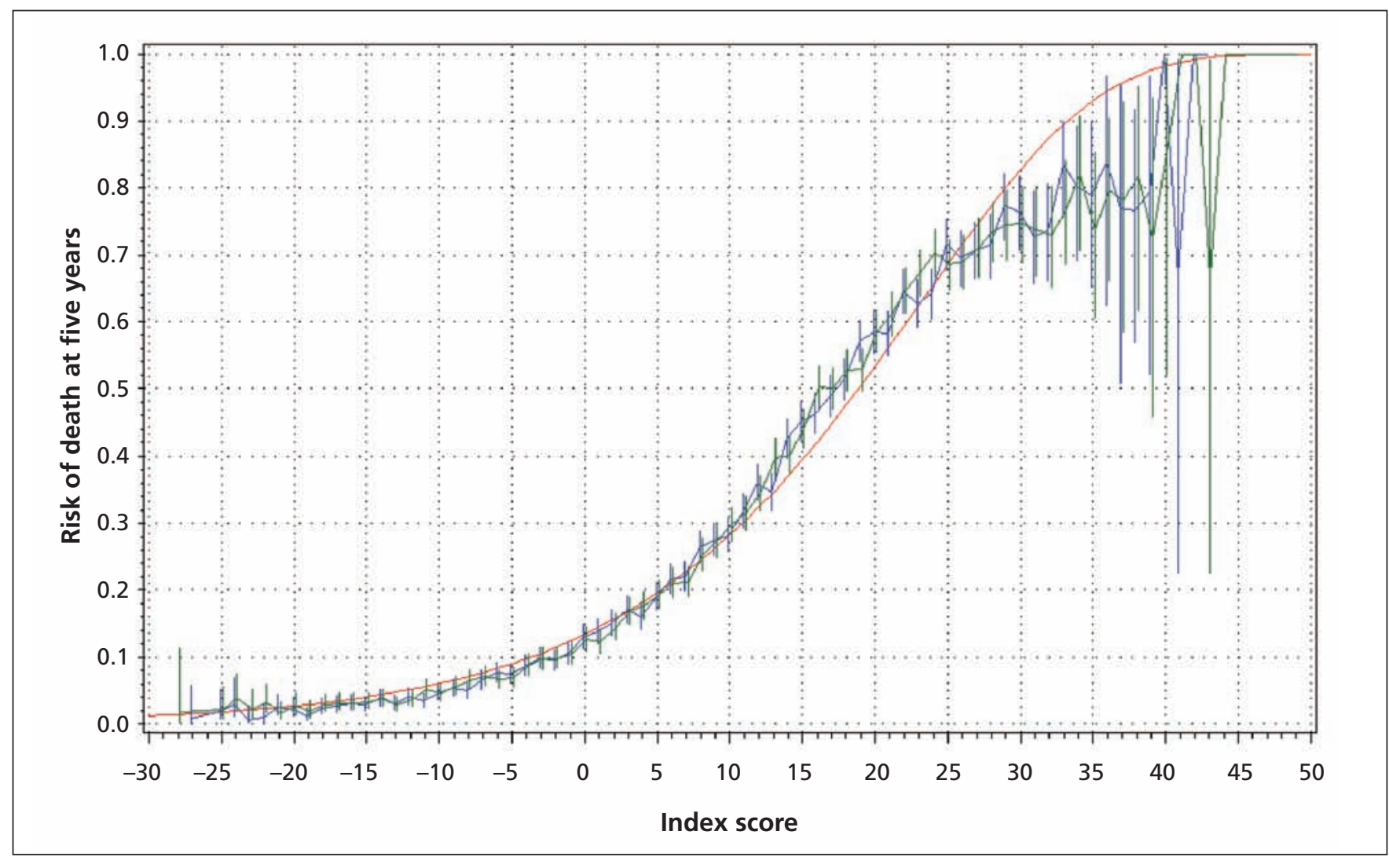

Figure 2: Comparison of predicted and actual risk of death at five years, by index score. The predicted (red line) and observed five-year mortality rates (vertical axis) in the derivation cohort (blue line) and validation cohort (green line) for each prognostic index score (horizontal axis) are shown. Vertical bars $=95 \%$ confidence intervals for the observed mortality rates.

\section{Strengths}

Our study has several notable strengths. First, we used a large, population-based data set that was collected using multiple checks for validity and has been used extensively for research. Second, the methods used to create our survival model were robust, including imputation for missing data, proper modeling of continuous variables ${ }^{20}$ and proper translation of the final model into a scoring system with expected outcomes. ${ }^{21}$ Third, we assessed the discrimination and accuracy of the index in a second group of patients that was not used to develop the index, thereby avoiding biased conclusions from over-fitting. Fourth, all of the variables required for our prognostic index are clear, objective and readily available to patients and clinicians. Fifth, our index is easy to apply and can be computed quickly and without a calculator using the point system outlined in Figure 1. In addition, we created a simple spreadsheet that will quickly calculate predicted survival for individual patients.

\section{Limitations}

The limitations of our analysis should also be noted. First, the model was derived from a group of patients who were deemed eligible for transplantation and placed on a waiting list. As such, the index should be applied only to patients who are considered to be candidates for renal transplantation. Frequently, this assessment is straightforward. In cases in which candidacy for transplantation is difficult to determine, transplant listing guidelines ${ }^{14,22}$ could be used to assess the applicability of this index to a particular patient. Second, the prognostic index showed the important influence of era or year of first renal replacement therapy on patient survival. As a result, the prognostic index will need to be updated occasionally, as recommended, ${ }^{23}$ when new data become available from the United States Renal Data System. However, since year was entered into the model as a linear variable (Appendix 3), this model is likely to be safely usable for years, up to 2011 as a short-term extrapolation.

Third, aside from donor type (i.e., living or deceased), we intentionally did not add donor characteristics to the model because we intended it to be used for pre-transplant decisionmaking. In many jurisdictions, older patients often receive older or "expanded criteria" donor kidneys. ${ }^{24}$ Since post-transplant survival is attributable in part to donor characteristics, the survival probabilities we have obtained reflect current practices of allocation. If major changes in allocation were to occur, such as ones based on "net benefit" (i.e., in which a donor kidney is given to the person with the greatest likelihood for improved survival), the probabilities we have derived may no longer be accurate. As such, the model should be updated after any major changes in allocation policy. Finally, information on comorbidities in the United States Renal Data System may be incompletely captured, and only 
the presence - not the severity - of the illness is accounted for in the model. Hence, the impact of some comorbidities on survival may be underestimated by our model. Prospective validation of the model would help determine if this underestimation is indeed happening.

\section{Conclusion}

Our prognostic index can provide important information for the prediction of mortality among patients with end-stage renal disease who are eligible for transplantation. We believe that this renal prognostic index can provide valuable quantitative survival data for clinicians and patients to use when discussing dialysis and transplantation treatment options.

\section{This article has been peer reviewed.}

Competing interests: None declared.

Contributors: All of the authors were involved in the conception and design of the study, the acquisition, analysis and interpretation of the data and the drafting and critical revision of the manuscript. All of them approved the final version of the manuscript submitted for publication. Carl van Walraven had full access to all of the data and takes responsibility for the integrity of the data and accuracy of the data analysis.

Acknowledgment: The authors thank the United States Renal Data System (USRDS) for providing data for analysis.

Funding: None.

\section{REFERENCES}

1. Wolfe RA, Ashby VB, Milford EL, et al. Comparison of mortality in all patient on dialysis, patients on dialysis awaiting transplantation, and recipients of a first cadaveric transplant. $N$ Engl J Med 1999;341:1725-30.

2. Laupacis A, Keown P, Pus N, et al. A study of the quality of life and cost-utility of renal transplantation. Kidney Int 1996;50:235-42.

3. Rabbat CG, Thorpe KE, Russell JD, et al. Comparison of mortality risk for dialysis patients and cadaveric first renal transplant recipients in Ontario, Canada. J Am Soc Nephrol 2000;11:917-22.

4. Hernandez D, Rufino M, Bartolomei S, et al. A novel prognostic index for mortality in renal transplant recipients after hospitalization. Transplantation 2005;79:337-43.

5. Jassal SV, Schaubel DE, Fenton SSA. Predicting mortality after kidney transplantation: a clinical tool. Transpl Int 2005; 18:1248-57.
6. Baskin-Bey ES, Kremers W, Nyberg SL. A recipient risk score for deceased donor renal allocation. Am J Kidney Dis 2007;49:284-93.

7. Wolfe RA, McCullough KP, Schaubel DE, et al. Calculating life years from transplant (LYFT): methods for kidney and kidney-pancreas candidates. Am J Transplant 2008;8:997-1011.

8. Royston P, Altman DG. Regression using fractional polynomials of continuous covariates: Parsimonious parametric modelling. Appl Stat 1994;43:429-67.

9. Sauerbrei W, Royston P. Building multivariable prognostic and diagnostic models: transformation of the predictors by using fractional polynomials. J R Stat Soc [Ser A] 1999;162:71-94.

10. Sauerbrei W, Meier-Hirmer C, Benner A, et al. Multivariable regression model building by using fractional polynomials: Description of SAS, STATA and R programs. Comput Stat Data Anal 2006;50:3464-85.

11. Newstead CG. Side effects of immunosuppression — what patients really think. Nephron Clin Pract 2004;97:c31-2.

12. Halloran PF. Immunosuppressive drugs for kidney transplantation. $N$ Engl J Med 2004;351:2715-29.

13. Vajdic CM, McDonald SP, McCredie MR, et al. Cancer incidence before and after kidney transplantation. JAMA 2006;296:2823-31.

14. Kasiske BL, Cangro CB, Hariharan S, et al. The evaluation of renal transplantation candidates: clinical practice guidelines. Am J Transplant 2001;1(Suppl 2):3-95.

15. Danovitch G, Savransky E. Challenges in the counseling and management of older kidney transplant candidates. Am J Kidney Dis 2006;47:S86-97.

16. Rao PS, Merion RM, Ashby VB, et al. Renal transplantation in elderly patients older than 70 years of age: results from the Scientific Registry of Transplant Recipients. Transplantation 2007;83:1069-74.

17. Meier-Kriesche HU, Port FK, Ojo AO, et al. Effect of waiting time on renal transplant outcome. Kidney Int 2000;58:1311-7.

18. United States Renal Data System (USRDS) annual report: Survival, mortality, and causes of death. Am J Kidney Dis 2003;41:S151-64.

19. Miskulin D, Bragg-Gresham J, Gillespie BW, et al. Key comorbid conditions that are predictive of survival among hemodialysis patients. Clin J Am Soc Nephrol 2009;4:1818-26.

20. van Walraven C, Hart R.G. Leave 'em alone - why continuous variables should be analyzed as such. Neuroepidemiology 2008;30:138-9.

21. Sullivan LM, Massaro JM, D'Agostino RB Sr. Presentation of multivariate data for clinical use: the Framingham study risk score functions. Stat Med 2004;23:1631-60.

22. Knoll G, Cockfield S, Blydt-Hansen T, et al. Canadian Society of Transplantation consensus guidelines on eligibility for kidney transplantation. CMAJ 2005; 173:1181-4

23. Toll D.B., Janssen K.J.M., Vergouwe Y., et al. Validation, updating and impact of clinical prediction rules: a review. J Clin Epidemiol 2008;61:1085-94.

24. Rao PS, Ojo A. The alphabet soup of kidney transplantation: SCD, DCD, ECD fundamentals for the practicing nephrologist. Clin J Am Soc Nephrol 2009;4: 1827-31.

\section{Correspondence to: Dr. Carl van Walraven, Clinical}

Epidemiology Program, Ottawa Hospital Research Institute,

Rm. ASB1-003, Ottawa Hospital, Civic Campus, 1053 Carling Ave.,

OttawaON K1Y4E9; carlv@ohri.ca
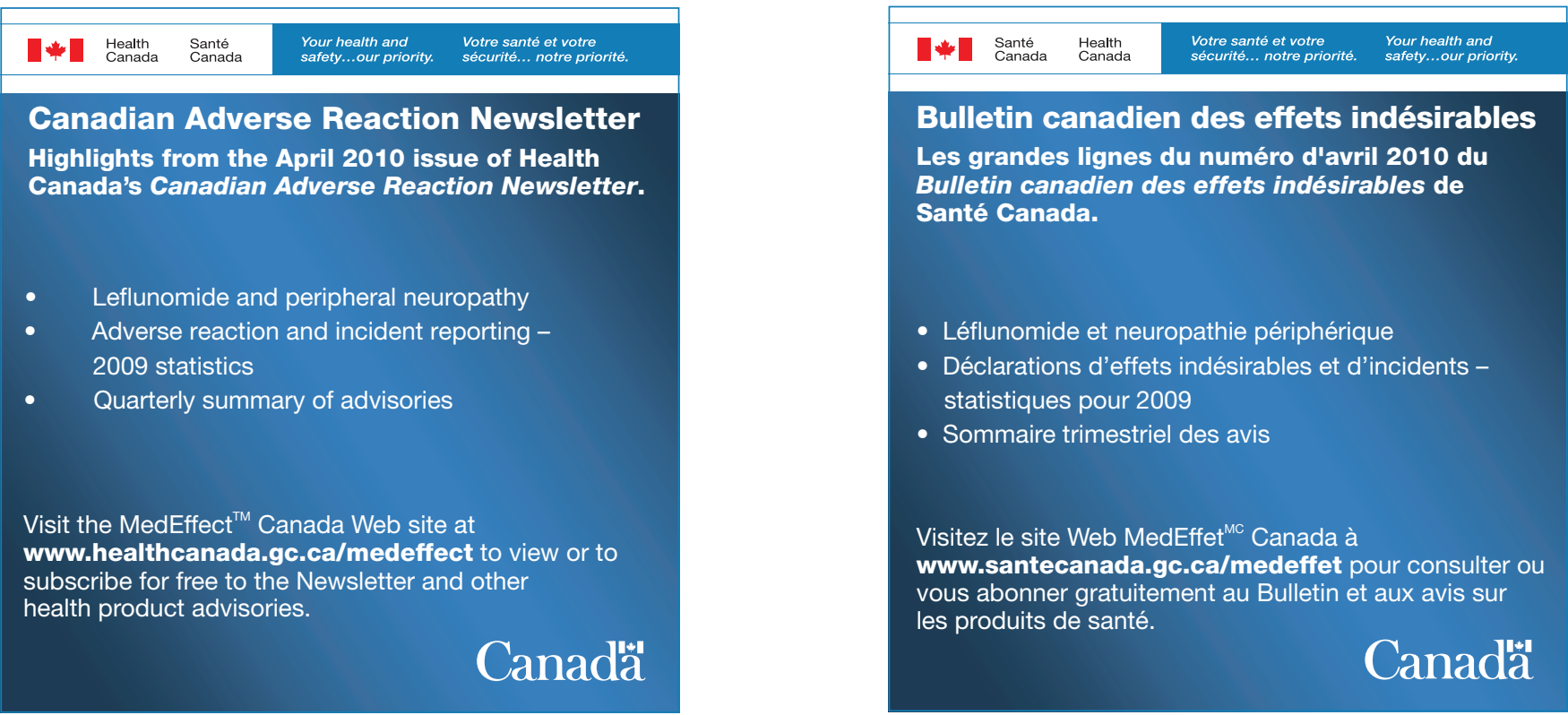